\title{
Early Surgical Intervention and Functional Outcome in Acetabulum Fracture
}

\author{
Keywords: Acetabulum fracture; Early intervention; Functiona \\ outcome
}

\begin{abstract}
Aim of the study: To analyse the relationship between early surgica intervention and post-operative functional outcomes in acetabular fracture incidences. Time to surgery is a predictor of radiological and functional outcome for both simple and complex patterns of fracture.

Methods: This is a cross sectional study done in a tertiary hospital for patient who have sustained acetabular fractures between January 2011 and December 2013 and had undergone surgical intervention,
\end{abstract} with a minimum follow-up duration of 1 year.

Results: This study involved 47 cases where the mean age was 35.6 years old. For simple fractures, an increase in the time to surgery of one day reduced on average, the Harris Hip Score by 1.5 points. Whilst for complex fractures, reduction in the Harris Hip Score averaged 1.87 points daily. When time was measured as a categorical variable, an anatomical reduction was more likely if surgery was performed within 15 days (simple) and 5 days (complex). An excellent/good functional outcome was more likely when surgery was performed within 8 days for both simple and complex fracture patterns.

Conclusions: The time to surgery is a significant predictor of radiological, functional outcome and post operative stay for both simple and complex displaced fractures of the acetabulum. The organisation of regional trauma services must be capable of satisfying these time-dependent requirements to achieve optimal patient outcomes.

\section{Introduction}

Patients with acetabular fractures have significant post-operative functional deficits as compared to other fractures. In many cases, anatomical reduction alone has shown good surgical outcome, however it was unable to achieve good functional outcome. There have been a number of cases noted with poor functional outcomes despite near vv reduction, most likely due to the initial chondral damage sustained at inju

ry. Many publications and studies on treatment of acetabular fractures and outcomes have been done, however the relation between outcome and early surgical intervention is still poorly evidenced and understood.

Surgical interventions for acetabular fractures vary and depend on many variables including the nature of the injury, fracture pattern, surgeon's experience, timing of surgery and rehabilitation. It is important to understand, and thus better to manage the factors within a surgeon's control in order to obtain the best possible functional and radiological outcomes. Poor fixation can lead to poor outcomes and hence affect patient's quality of life.

It is an unfortunate fact that the healthcare system in Malaysia has little or no useful information on the incidences of acetabular

\section{Journal of}

Orthopedics \& Rheumatology

\section{Gunaseelan $\mathbf{P}^{1}$, Aswadi A', Thevarajan $\mathrm{K}^{2}$, Ong $\mathbf{Z W}^{2}$, Norhafizah $\mathbf{M}^{\mathbf{2}}$ and Abdul Halim $\mathbf{R}^{\mathbf{1}}$}

${ }^{1}$ Department of Orthopaedics, Faculty of Medicine, University Kebangsaan Malaysia

${ }^{2}$ Department of Orthopaedics, Hospital Sultanah Aminah, Johor Malaysia

*Address for Correspondence

Gunaseelan P, Department of Orthopaedics, Faculty of Medicine, University Kebangsaan Malaysia, E-mail: gunaseelandr@gmail.com

Submission: 19 May, 2016

Accepted: 17 August, 2016

Published: 22 August, 2016

Copyright: $\odot 2016$ Gunaseelan P, et al. This is an open access article distributed under the Creative Commons Attribution License, which permits unrestricted use, distribution, and reproduction in any medium, provided the original work is properly cited.

fractures due to the limited interest in the subject. In most cases, inadequate data collection and when collected, the data is often incomplete, inaccurate, not well documented and based on outdated coding systems. In addition, the outcome of treatment is unknown as follow-ups are irregular and non specific. However, this appears to be the case in most countries in the Asian region. As such, references for this study have been primarily sourced from UK and America. Our aim is to analyse the relationship between early surgical intervention and post-operative functional outcomes in acetabular fracture incidences.

\section{Materials and Methods}

This is a cross sectional study on patients, who have undergone acetabular surgery. Data was collected from case notes including post-operative notes, nursing reports, vitals charts and clinic followup notes.

The inclusion criteria for this study was any patient treated in Hospital Sultanah Aminah Johor Bahru, who had sustained acetabular fracture between January 2011 and December 2013 and had undergone surgical intervention, with a minimum follow-up period of 1 year.

Exclusion criteria for this study is any patient with congenital or developmental acetabular dysplasia, previous fractures involving the acetabular region and spinal cord injury patients who have undergone previous acetabular surgery. Fracture characterization was conducted using standard pre-operative radiographs (anteroposterior pelvis, inlet \& outlet views of the pelvis, and Judet's view) and where required, computed tomography (CT). All surgical procedures were performed by two senior orthopaedic surgeons with experience of 8 to 10 years.

Post-operatively, patients were monitored either in a critical care unit (CCU) or an orthopaedic ward based on physiological parameters as assessed by the anaesthetists. All patients were given antithrombotic prophylaxis against deep vein thrombosis (DVT). 
Citation: Gunaseelan P, Aswadi A, Thevarajan K, Ong ZW, Norhafizah M, et al. Early Surgical Intervention and Functional Outcome in Acetabulum Fracture. J Orthopedics Rheumatol. 2016; 3(1): 5.

When patients attained satisfactory ambulatory status, they were discharged home or to an outpatient rehabilitation facility. Following discharge, patients were reviewed at 6 weeks, 3 months, 6 months and 12 months. At each outpatient review, standard radiographs of the pelvis were repeated, and CT scans were repeated only in the event of complications.

Acetabular fracture fixation techniques were critically reviewed using a modified version of the criteria described by Matta et al. This involved the summation of the fracture displacement on 2 (two) plain radiographic views. These values were obtained for both preoperative and post-operative radiographs (Grading of Heterotopic Ossification based on Brooker Classification).

Pre-operative factors studied include patient's age and gender, mechanism of injury, fracture classification, associated injuries and time to surgical intervention. Post-operative factors reviewed included duration of stay in CCU, complications and ambulatory status at the most recent follow-up.

Functional outcome of patients with acetabular fractures were graded using the Harris Hip scoring system. Functional outcome scores were correlated to the time of surgery and some pre-operative and post-operative factors using the Chi-squared test at $5 \%$ level of significance.

\section{Results}

\section{Demographic}

53 incidences of acetabular fractures were recorded between 2011 and 2014. Of which, 6 patients were not included in further analysis due to incomplete data. Of the 47 patients analysed, 33 (70.2\%) were males and 14 (29.8\%) were females, ranging from 19 to 53 years of age with a mean age of 35.6. Twenty eight (59.6\%) patients were ethnic Malay, followed by 13 (27.7\%) ethnic Indians and $6(12.8 \%)$ ethnic Chinese. Thirty four (72.3\%) patients had right acetabular fractures whilst $13(27.7 \%)$ patients had left acetabular fractures. Most patients $(87.2 \%)$ suffered acetabular fractures due to motor vehicle accidents, followed by patients falling from a height, and sustaining a sport injury. Based on Letournel \& Judet's classification, 28 fractures (59.6\%) were simple, and 19 complex (40.4\%), with the distribution of fracture and surgical approach as detailed in Table 1.

\section{Analyzing waiting time for surgery and post operative stay}

The time period between trauma and surgical intervention ranged from a minimum of 3 days, and a maximum of 20 days with a median of 9 days. Data analysis noted no significant association between fracture type $(p=0.31)$ and gender $(p=0.20)$. The numbers of patients operated on in broad interval categories for both simple and complex acetabulum fracture are detailed in Table 2 and Figures 1 and 2.

Post operative stay, data shows that distribution of days of admission in subjects in a boxplot diagram, the minimum is 3 days; the maximum is 14 days with a median of 7 days. Data analysis noted that, there is no significant association between post operative and gender $(\mathrm{p}=0.56)$.

Using Mann-Whitney test, $\mathrm{p}<0.01$, there is a highly significant association between fracture type and post operative stay. Post operative stay is significantly shorter for patients with simple fracture (average of 5.8 days) as compared to patients with complex fractures (average of 8.2 days). The numbers of patients in broad post operative stay interval categories for both simple and complex acetabulum fracture are detailed in Table 3.
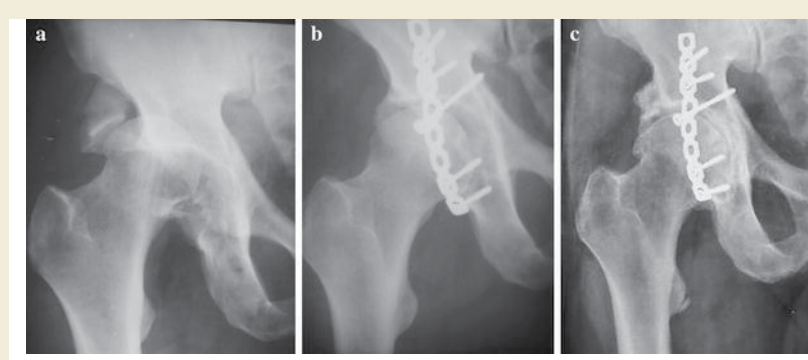

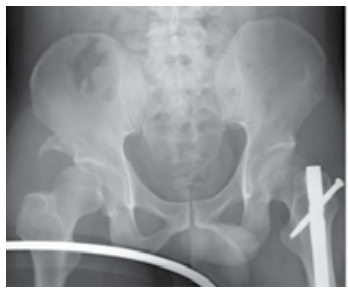

(A) Pre - operative

Figure 1: Simple fracture.

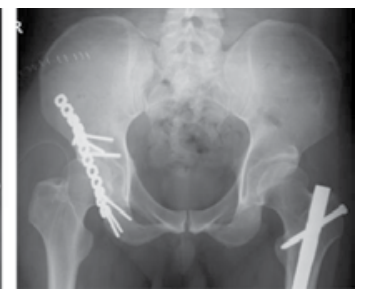

(B) Post - operative

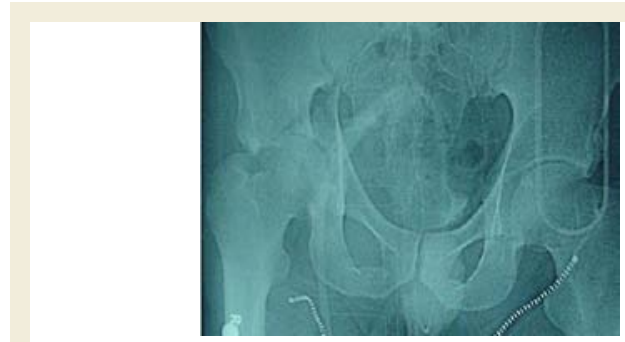

(A) Pre - operative

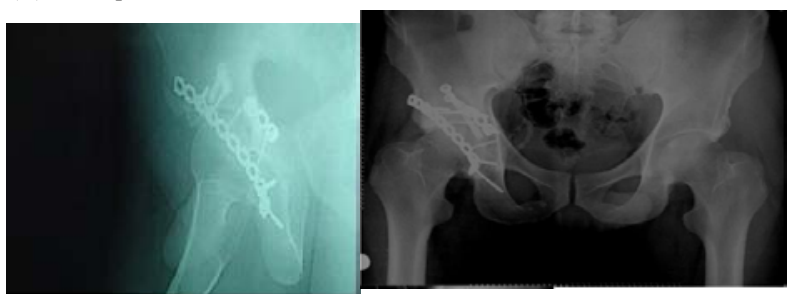

(B) Post - operative

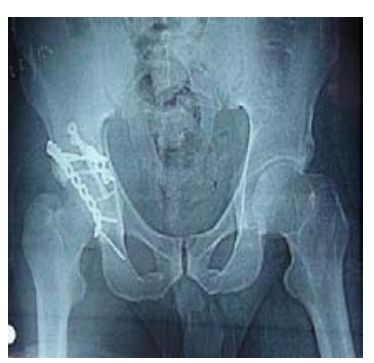

(C) 1 year follow up

Figure 2: Complex fracture. 
Citation: Gunaseelan P, Aswadi A, Thevarajan K, Ong ZW, Norhafizah M, et al. Early Surgical Intervention and Functional Outcome in Acetabulum Fracture. J Orthopedics Rheumatol. 2016; 3(1): 5.

\begin{tabular}{|c|c|c|c|c|c|}
\hline & Total & $\%$ & & Total & $\%$ \\
\hline Simple fracture types & 28 & $60 \%$ & Complex fracture types & 19 & $40 \%$ \\
\hline Anterior column & 3 & $11 \%$ & $\begin{array}{l}\text { Posterior wall + posterior } \\
\text { column }\end{array}$ & 6 & $32 \%$ \\
\hline Posterior wall & 12 & $43 \%$ & Anterior + posterior column & 3 & $16 \%$ \\
\hline Posterior column & 4 & $14 \%$ & $\begin{array}{l}\text { Anterior column }+ \text { posterior } \\
\text { hemitransverse }\end{array}$ & 4 & $21 \%$ \\
\hline Transverse & 8 & $29 \%$ & Transverse and posterior wall & 4 & $21 \%$ \\
\hline Anterior wall & 1 & $4 \%$ & T-shaped & 2 & $11 \%$ \\
\hline $\begin{array}{l}\text { Surgical approach } \\
\text { used }\end{array}$ & Total & & & & \\
\hline $\begin{array}{l}\text { Kocher-Langenbeck } \\
(\mathrm{KL})\end{array}$ & 25 & & & & \\
\hline Ilioinguinal (IL) & 12 & & & & \\
\hline Triradiate & 4 & & & & \\
\hline Combined $\mathrm{KL}$ and $\mathrm{IL}$ & 5 & & & & \\
\hline Extended iliofemoral & 1 & & & & \\
\hline
\end{tabular}

Table 1: The distribution of fracture types and the surgical approach used (number of patients and percentage).

between patients operated within 1 to 7 days $(\mathrm{p}=0.001)$ and between 8 to 14 days $(\mathrm{p}=0.02)$.

(The longer we wait for surgery leads to difficult reduction of fracture due to callus formation, more soft tissue manipulation, more bleeding, longer operative time, longer GA complication and delay in mobilizations, this leads to longer post operative stay compared to early surgical intervention. Stable patient is important but there must not be prolonged till more than 1 week, due to above reason they due to stay longer in hospital)

\section{Analyzing time to surgery and functional outcome}

Functional: Functional outcomes for all patients were assessed 6 weeks, 3 months, 6 months and 1 year after surgery using the Harris Hip Score. Functional outcomes showed significant improvements in subsequent clinical follow ups after surgery, as shown in Chart 1. Patients with excellent/good functional outcome increased from $23.4 \%$ (11 patients) at 6 weeks to $74.5 \%$ of patients (35) after 1 year.

Analysis of the relationship between functional score and waiting time to surgery showed a highly significant association for all results assessed at 6 weeks, 3 months, 6 months and 1 year. $(p<0.001)$ and both simple and complex fracture types. Functional scores were analysed using the Mann-Whitney test on simple fracture types and complex fracture types. Patients with a shorter waiting time to surgery ( $<15$ days) showed higher incidences of Excellent/Good functional outcomes, whilst patients with longer waiting times $(>15$ days) showed significantly poorer functional outcomes for simple and complex fracture types respectively.

Interesting to note, excellent functional outcomes were achieved only by patients who were operated on within 8 days for both simple and complex type fractures. Following which, functional outcome results steadily decreased to Good and Fair or Poor with longer waiting times.

The scatter plot diagram in Chart 2 shows the distribution of functional outcomes against the waiting time to surgery for simple and complex fracture types respectively. In both instances, there is strong negative correlation $(-0.77$ for simple fracture types and -0.81 for complex fractures) between the functional outcomes of patients and the waiting time to surgery.

The distribution of patients' functional outcomes vs. waiting time to surgery was further analysed using the median of range for each Harris Hip Score outcome category considering the minimum score of 40 . Assuming a linear relationship $\left(\mathrm{R}^{2}=0.59\right.$ and $\mathrm{R}^{2}=0.64$ for simple and complex fracture types respectively), a significant average daily reduction in the Harris Hip Score results (1.5 and 1.87 points for simple and complex fracture types respectively) per additional day of waiting time to surgery was noted.

Analysis of the association between functional score and gender, age and fracture time at 6 weeks, 3 and 6 months and 1 year noted no significant association.

Table 2: Waiting time to surgery intervals by patterns of fracture.

\begin{tabular}{|l|r|r|r|r}
\hline Time period (days) & \multicolumn{1}{|c|}{$<$ days } & $\mathbf{8}$ to 14 days & $>14$ days & \multicolumn{1}{|c}{ Total } \\
\hline Simple fracture & & & & \\
\hline Complex fracture & 14 & 10 & 4 & 28 \\
\hline
\end{tabular}

Table 3: Post operative length of stay by patterns of fracture.

\begin{tabular}{|l|r|r|r|r}
\hline Time period (days) & \multicolumn{1}{|c|}{$<$ days } & 6 to 10 & $>10$ days & \multicolumn{1}{c}{ Total } \\
\hline Simple fracture & 17 & 9 & 2 & 28 \\
\hline Complex fracture & 5 & 10 & 4 & 19 \\
\hline
\end{tabular}

Table 4: The radiological criteria post surgery based on the gap remaining at fracture site after reduction types.

\begin{tabular}{|c|c|c|c|}
\hline Reduction & Gap & Simple & Complex \\
\hline Anatomic & $0-1 \mathrm{~mm}$ & 22 & 9 \\
\hline Adequate & $2-3 \mathrm{~mm}$ & 4 & 3 \\
\hline
\end{tabular}




\section{Analyzing time to surgery and radiological outcome}

Radiological: Radiological outcomes for all patients were assessed 6 weeks, 3 months, 6 months and 1 year after surgery using the Matta radiological scoring method. Radiological scores showed significant reduction in subsequent clinical follow ups after surgery. Patients with excellent/good functional outcome decreased from 100\% (47 patients) at 6 weeks to $52.5 \%$ of patients (35) after 1 year. However, the reduction gap by plain radiographic imaging taken at 6 weeks (Table 4) showed poor results for 9 patients and adequate results for 7 patients indicating reduction gap measurements are better able to predict radiological outcomes in patients over the longer term.

Analysis of the relationship between radiological score and waiting time to surgery showed a highly significant association for all results assessed at 3 months, 6 months and 1 year ( $<<0.001$ for simple and $\mathrm{p}<0.002$ for complex fracture types). Radiological scores were analysed using the Mann-Whitney test on simple fracture types and complex fracture types. Patients with a shorter waiting time to surgery ( $<15$ days) showed higher incidences of Excellent/Good radiological outcomes, whilst patients with longer waiting times (>15 days) showed significantly poorer radiological outcomes for simple and complex fracture types respectively similar to functional outcome results, excellent radiological outcomes were achieved only by patients who were operated on within 8 days for both simple and complex type fractures. Following which, radiological outcome results steadily decreased to Good and Fair or Poor with longer waiting times. Analysis of the association between radiological score and gender, age and fracture time at 6 weeks, 3 and 6 months and 1 year noted no significant association.

\section{Analyzing time to surgery and complication}

Complications: The occurrence of local and systemic complications for both types of fractures are for early and late complications developed against waiting time to surgery. Patients operated on within 7 days are significantly less susceptible to early and late complications for both fracture types. Patients with complex fractures are more prone to developing late complications if waiting time to surgery exceeds 14 days.

\section{Discussion}

The aim of the treatment for displaced fractures of the acetabulum is to obtain an accurate and stable anatomical reduction as well as a functional, mobile and painless hip joint [1]. It has been well documented that the accuracy of reduction correlates with outcome and with a reduced risk of post-traumatic osteoarthritis of the hip [2]. Delay in reduction and definitive fixation of these fractures results in an increase in the formation of scar tissue between bony fragments and the formation of early callus at the fracture site. In these circumstances the surgeon will be faced with a more difficult exposure of less mobile fracture fragments, which are harder to reduce [2]. Techniques of indirect reduction are less likely to be effective. The reduced likelihood of obtaining an accurate and stable anatomical reduction will lead to a poor outcome. Delay in fixation may also be detrimental to the viability of the femoral head in cases of persistent subluxation [3]. There is an increase in the incidence of both chondrolysis and osteonecrosis of the head with delayed reconstruction [3].
The timing of surgery in relation to displaced fractures of the acetabulum has been reported in related literature. Johnson et al. described a rate of anatomical reduction of only $52 \%$ in fractures which were operated on more than 21 days from the time of injury, and recently Griffin, Beaule and Matta found that for complex fracture types, the accuracy of reduction and the clinical results correlated significantly but the time to surgery did not $[4,5]$.

Various authors have categorized time to surgery to assess the relationship of delays in surgery and outcome. Mears et al.categorized time periods into less than two days from injury, 3 to 10 days and 11 to 21 days [6]. They reported that patients with a delay of more than 11 days had a significantly lower rate of anatomical reduction. Matta divided the time to surgery into three periods: 1 to 7 days, 8 to 14 days and 15 to 21 days [5]. Of the 47 patients in this study, there was a significant difference in the quality of the reduction obtained when comparing patients operated on days 1 to 14 with those undergoing fixation at more than 15 days.

Previous studies have described the effect of a delay in surgery for displaced fractures as a whole group and only measured time as a categorical variable. In this study we assessed the effect of the time to surgery for both simple and complex types individually, and the category 'time' was treated as both continuous and categorical variables.

Our results when analyzing time to surgery show that for both patterns of fracture, the odds of obtaining an anatomical reduction and an excellent/good functional outcome reduce significantly as the time to surgery increases. Excellent/good functional outcomes were achieved mostly by patients who were operated on within the first 15 days of injury for both simple and complex fractures.

Matta also drew attention to other factors which influence the outcome of patients with displaced fractures of the acetabulum, such as injuries of the femoral head, advanced patient age, operative complications and other bodily injury [5].

Murphy et al. studied prognostic factors and their relationship to the functional outcome of fractures of the acetabulum [7]. He identified four dominant prognostic factors which were associated with a suboptimal outcome: complex fracture type, imperfect reduction, the presence of local complications, and heterotopic bone. Factors which were not significant were gender, palsy of the sciatic nerve and dislocation of the hip. The clinical results in patients with associated injuries have been reported to be similar to those with an isolated fracture $[8,9]$. In our study, age, gender, and type of fracture and early complication had no significant effect on functional outcome.

The accuracy of reduction is thought to be strongly related to the fracture type. Mears et al. showed in his study of 424 fractures treated by operation, that simple fractures were reduced anatomically in $87 \%$ of patients, whereas associated fractures could be reduced anatomically in only 59\% [8]. Matta had similar results, achieving anatomical reduction in $96 \%$ of simple fractures and only $64 \%$ of associated fractures [5]. The quality of reduction is also considered to be related to the timing of surgery. Mears et al. found that if surgery was delayed for more than 11 days after injury, there were significantly fewer anatomical reductions [8]. We also found the same relationship 
Citation: Gunaseelan P, Aswadi A, Thevarajan K, Ong ZW, Norhafizah M, et al. Early Surgical Intervention and Functional Outcome in Acetabulum Fracture. J Orthopedics Rheumatol. 2016; 3(1): 5.

ISSN: $2334-2846$

between radiological score and waiting time to surgery. In addition, the reduction gap by plain radiographic imaging showing reduction gap measurements are better able to predict radiological outcomes in patients over the longer term.

More recently, Plaisier et al. reviewed 100 patients treated surgically for pelvis or acetabulum fractures and reported that fixation within 24 hours of injury was associated with lower mortality, shorter hospital stay, and less organ failure [3]. Other reports regarding the timing of acetabulum fracture fixation have focused on better quality of reduction and function with early fixation, ranging from 5 to 11 days $[10,11]$. All of these studies are limited by relatively small numbers of patients with heterogeneous spectrum of associated injuries and types of treatment. Based on our data, it shows postoperative stay is significantly shorter for patients with simple fracture (average of 5.8 days) as compared to patients with complex fractures (average of 8.2 days).

There is a significant association between the type of fracture and late complications. Patients with complex fracture are more likely to develop late complications such as Heterotopic ossification (HO), Avascular necrosis (AVN) and Osteoarthritis (OA), as compared to patients with simple fractures. This may be attributed to the incidence of AVN described in published papers varies from $3 \%$ to $53 \%[12,13]$. Base on our study AVN rate significantly increased in patients with prolonged time of surgery and patients sustaining a posterior fracture dislocation of the hip, the incidence of AVN more common. These findings are consistent with those reported in the literature $[7,13]$. The overall incidence of OA following operatively treated acetabular fractures was $100 \%$ for patient with complex fractures at 1 year follow [14]. This emphasises Matta's view that "the primary complication following a fracture of the acetabulum is post-traumatic osteoarthritis" [5]. However, Matta described rates of OA as high as $46 \%$ [5]. Patient who developed HO, in our study had a higher incidents of $\mathrm{HO}(36 \%)$, which may be due to soft tissue handling during surgery. Meta-analysis showed an incidence of $25.6 \%$ of HO following operation for acetabular fractures.

The strengths of our study are the large number of patients in a single institution, experience surgeons, the long follow-up (88.6\%) duration of 1 year; review of the patients to an agreed protocol, control of potential confounding variables, the categorisation of time periods, the frequency of delay to surgery and the analysis of results for both simple and complex types of fracture.

It is worth noting that 30 of the 47 patients (63.8\%) were referred to our institution from other hospitals and constituents. The main cause of delays was the lack of available beds disallowing unrestricted transfer to our hospital. Delay in initiating referral was also a contributing factor. The majority of the hospitals in the southern region of Malaysia do not provide pelvic and acetabulum trauma surgeries. Bircher and Giannoudis highlighted the shortcomings of the pelvic and acetabulum trauma service in the United Kingdom as a contributing factor to delays in surgery [15].

\section{Conclusion}

The odds of achieving an anatomical reduction and an excellent/ good functional outcome reduces significantly for both simple and complex displaced fractures of the acetabulum as the time to surgery increases. Delays in definitive fixation have more effect on outcome for the complex fracture subgroup. For management to be effective, definitive surgery should be performed as soon as possible. Regional and national trauma referral systems must be capable of achieving time dependent targets to optimize patient outcome.

\section{References}

1. Laird A, Keating JF (2005) Acetabular fractures: a 16-year prospective epidemiological study. J Bone Joint Surg Br 87: 969-973.

2. Mucha P Jr, Farnell MB (1984) Analysis of pelvic fracture management. J Trauma 24: 379-386.

3. Pohlemann T, Tscherne H, Baumgartel F, Egbers HJ, Euler E, et al. (1996) Pelvic fractures: epidemiology, therapy and long-term outcome: overview of the multicentre study of the Pelvis Study Group. Unfallchirurg 99: 160-167.

4. Giannoudis PV, Grotz MR, Papakostidis C, Dinopoulos H (2005) Operative treatment of displaced fractures of the acetabulum: a meta-analysis. J Bone Joint Surg $\mathrm{Br}$ 87: 2-9.

5. Matta JM (1996) Fractures of the acetabulum: accuracy of reduction and clinical results in patients managed operatively within three weeks after the injury. J Bone Joint Surg Am 78: 1632-1645.

6. Letournel E, Judet R (1993) Fractures of the acetabulum. $2^{\text {nd }}($ edn.), SpringerVerlag Berlin Heidelberg, pp. 29-62.

7. Murphy D, Kaliszer M, Rice J, McElwain JP (2003) Outcome after acetebular fractures. Prognostic factors and their inter-relationships. Injury 34: 512-517.

8. Mears DC, Velyvis JH, Chang CP (2003) Displaced acetabular fractures managed operatively: indicators of outcome. Clin Orthop Relat Res 173-186.

9. Deo SD, Tavares SP, Pandey RK, El-Saied G, Willett KM, et al. (2001) Operative management of acetabular fractures in Oxford. Injury 32: 581-586.

10. Judet R, Judet J, Letournel E (1964) Fractures of the acetabulum: classification and surgical approaches for open reduction. Preliminary report. J Bone Joint Surg Am 46: 1615-1646.

11. Letournel E (1980) Acetabulum fractures: classification and management Clin Orthop Relat Res 81-106.

12. d'Aubigné RM, Postel M (1954) Functional results of hip arthroplasty with acrylic prosthesis. J Bone Joint Surg Am 36-A: 451-475.

13. Johnson EE, Matta JM, Mast JW, Letournel E (1994) Delayed reconstruction of acetabular fractures 21-120 days following injury. Clin Orthop Relat Res 20-30.

14. Griffin DB, Beaule PE, Matta JM (2005) Safety and efficacy of the extended iliofemoral approach in the treatment of complex fractures of the acetabulum. J Bone Joint Surg Br 87: 1391-1396.

15. Bircher M, Giannoudis PV (2004) Pelvic trauma management within the UK a reflection of a failing trauma service. Injury 35: 2-6. 